\title{
Siglec10 as an Immunosuppressive and Tumorgenesis Signature for Prediction of Survival Prognosis in Gliomas
}

\section{Yanyan Feng}

Beijing University of Chinese Medicine

\section{Yuxiang Zhang}

Capital Medical University Sanbo Brain Hospital

\section{Ting Wang}

Beijing University of Chinese Medicine

\section{Yuyin Feng}

Beijing University of Chinese Medicine

\section{Hesong Wang}

Beijing University of Chinese Medicine

Kai Yuan ( $\nabla$ yuankai@bucm.edu.cn )

Beijing University of Chinese Medicine https://orcid.org/0000-0001-7239-449X

Primary research

Keywords: Siglec10, prognosis, glioma, mechanism, biomarker

Posted Date: July 14th, 2020

DOI: https://doi.org/10.21203/rs.3.rs-40997/v1

License: () (1) This work is licensed under a Creative Commons Attribution 4.0 International License. Read Full License 


\section{Abstract}

\section{Background}

Glioma is a type of tumor occurring in central nervous system. Specific factors mutations and molecular abnormalities are used to conduct glioma classification and clinical decision in recent decade. Siglec10 is a member of sialic acid binding immunoglobulin superfamily.

\section{Methods}

We analyzed the siglec10 expression in glioma patients with immunohistochemical (IHC) staining method and evaluated the survival prognosis. Bioinformatic datasets including TCGA and CGGA were used to validate the $\mathrm{IHC}$ results. Functional enrichment analysis including GO, KEGG, and GSEA were conducted to study the related mechanism of siglec10, either.

\section{Results}

High siglec10 expression had shorter survival prognosis than low siglec10 expression in patients especially with malignant gliomas. Bioinformatic datasets including TCGA and CGGA validated the IHC results and discovered the expression of siglec10 was higher in malignant subtype than benign subtype of gliomas. So, siglec10 contributes to the poor prognosis of gliomas. Furthermore, the related mechanisms of siglec10 in gliomas were investigated by functional enrichment analysis including GSEA, GO, and KEGG analysis. Siglec10 was correlated with inflammatory mediators, inflammatory cells, and inflammatory pathways in gliomas.

\section{Conclusions}

Siglec10 might take part in the immune response in tumor microenvironment to induce the progression and metastasis of glioma. This study showed siglec 10 was a biomarker in glioma and it might be the potential target of glioma immunotherapy in the future.

\section{Background}

Gliomas are responsible for $24 \%$ primary brain and central nervous system tumors. With the criteria of World Health Organization (WHO), gliomas are divided into four grades from WHO grade I to WHO grade IV (1). Low grade gliomas (WHO grade I, II) are regarded as benign tendencies with better prognosis survival for patients, while high grade gliomas (WHO grade III, IV) are considered as malignant tendencies with poor prognosis survival for patients (2). Gliomas also could be classified by histological features including oligodendroglioma, astrocytomas, ependymomas, etc (3). In recent decade, specific factors mutations and molecular abnormalities are used to conduct glioma classification and clinical decision (4). A specific gene mutation could be used to clarify low-grade or high-grade tumors. Except for classification, specific factors mutations and molecular abnormalities are also correlated with prognosis survival and immunotherapies of glioma patients. The predictive and prognostic biomarkers could be 
coexisted in glioma patients (5). Isocitrate dehydrogenase (IDH) is an enzyme to catalyze the oxidative decarboxylation of isocitrate. IDH mutation was found in low grade gliomas and correlated with better survival prognosis compared with IDH wide type patients (6). MGMT (0-6-methylguanine-DNA methyltransferase) is a DNA repair gene (7). MGMT methylation is a useful biomarker to predict the chemotherapy response of gliomas (8). So, it is important to discover and identify novel biomarker in glioma to predict the survival prognosis and therapy response of glioma patients.

Siglec10 (Sialic acid-binding Ig-like lectin 10) is a member of sialic acid binding immunoglobulin superfamily (9). Siglec10 bears an immunoreceptor tyrosine based inhibitory motif in the cytoplasmic domain. It is a ligand for CD52, which is the target of monoclonal antibody alemtuzumab (10). In addition, siglec10 also binds co-stimulatory molecule CD24 and vascular adhesion protein 1 (VAP-1) (11). Previous studies have investigated the functions and mechanisms of siglec10 in tumors. Zhang et al. found siglec10 decreased survival prognosis of hepatocellular carcinoma (12). The expression of siglec10 in tumor tissue was higher than the adjacent normal tissue. Barkal et al demonstrated tumor associated macrophages (TAM) express high siglec10 level in many types of tumors. Genetic deletion of siglec10, or the ligand CD24 could increase the phagocytosis of CD24 expressed tumors (13). BandalaSanchez et al. revealed siglec10 was increased in activated $C D 4^{+} T$ cells of humans (14). However, the function of siglec10 in gliomas has not been discovered.

In this study, we explored the expression and functions of siglec10 in gliomas. Firstly, we analyzed the siglec10 expression in glioma patients with immunohistochemical staining method and evaluated the survival prognosis of siglec10 in gliomas. Secondly, we used TCGA and CGGA datasets to validate the survival prognosis of siglec10 and discover the expression of siglec10 in different subtypes. Later, we tried to clarify the immunological mechanism of siglec10 in gliomas. Lastly, GO, KEGG, and GSEA analysis were used to conduct functional enrichment analysis of siglec10. In this study, we aimed to provide evidence of siglec10 as a predictive biomarker for glioma patients.

\section{Method}

\subsection{Patients and samples}

We enrolled 162 samples of glioma patients from Sanbo Brain Hospital Capital Medical University. The samples of gliomas with surgery were collected. The laboratory examination, clinical characteristics, and histological examination were used to diagnose glioma patients. Two pathologists evaluated the grades, subtypes, and molecule features of glioma samples. The information of follow-up glioma patients after surgery was obtained. In this study, the index overall survival (OS) which is a useful parameter of survival prognosis was used to define the end point of this study. Every glioma patient was required to sign the informed consent to study tissue in medical research. This study was approved by the Ethics Committee in the hospital (No.SBNK-2018-003-01). 
After obtaining the glioma samples, siglec10 expression in the samples was discovered by immunohistochemical staining method. Firstly, we baked the formalin fixed paraffin embedded slides for 4 hours. Secondly, we deparaffinated the slides and dehydrated with gradient ethanol. Then, we conducted antigen retrial the slides with citrate buffer, cyclooxygenase block, and antigen block. Furthermore, the slides were incubated with primary antibody rabbit anti-human siglec10 (abcam) overnight at $4{ }^{\circ} \mathrm{C}$. After first day incubation, second antibody horseradish peroxidase (HRP)-labeled goat anti-mouse and rabbit was used to incubate the slide with 1 hour. After second antibody incubation, 3'diaminobenzidine $(D A B)$ reagent was used to stain the slide and hematoxylin was used to counterstaine with the slide. At last, the slides were estimated by ImageJ software to analyze the siglec10 expression by 2 scientists. The cutoff value was evaluated by R.

\subsection{Siglec10 expression bioinformatics analysis}

Firstly, The Cancer Genome Atlas (TCGA) dataset (https://portal.gdc.cancer.gov) was used to conduct the bioinformatics analysis. TCGA was a powerful dataset to determine genetic mutations with method of genome sequence and bioinformatics in cancers. The value of fragments per kilobase per million mapped reads (FPKM) in gliomas was normalized. RNA sequence normalized datasets were regarded as input. Furthermore, the mRNA data from Chinese Glioma Genome Atlas (CGGA) to 2019 October was downloaded. CGGA is a useful dataset storing more than 2,000 brain tumor samples. In CGGA dataset, the data contain mRNA microarray, mRNA sequencing, whole exome sequencing, microRNA microarray, and related patient clinical characteristics. The different between groups were estimated by SPSS20.0.

In addition, the difference between glioma tissue and normal tissue in siglec10 expression was performed in Gene Expression Profiling Interactive Analysis (GEPIA) dataset. GEPIA is a powerful website performing analysis about gene expression profile in cancer and normal tissue.

\subsection{Functional enrichment analysis}

In this study, Gene Ontology (GO) and Kyoto Encyclopedia of Genes and Genomes (KEGG) analysis were used to conduct functional enrichment analysis. The spearman method was conducted to calculate the relationship between siglec10 and related genes. We filtered the genes with correlation >0.6. GO analysis was a powerful bioinformatics tool to determine cellular components (CC), molecular functions (MF), and biological process (BP). KEGG is a useful datasets collection tackling genomes, biological pathways, and diseases. At last, the database for annotation, "enrichplot" package, and "clusterProfiler" package were conducted to visualize the results. Apart from GO and KEGG analysis, Gene Set Enrichment Analysis (GSEA) was also used to discover the mechanism of siglec10 in glioma patients. GSEA could be conducted to investigate the biological mechanisms of genes. The relationship between immune cells infiltration and siglec10 was conducted by single sample GSEA (SSGSEA). The related data was extracted from PMID: 24138885. ssGSEA is an extension of GSEA evaluating specific gene set enrichment scores. ssGSEA transforms to gene set enrichment profile, leading to identify biological processes. The relationship between immune related gene sets and siglec10 was performed in the bioinformatic website (https://www.immport.org/). 


\subsection{Statistical analysis}

We used spearman $\chi^{2}$ test and $t$ test to conduct the statistical analysis. The survival prognosis was evaluated by Log-rank test. The hazard ration (HR) and 95\% confidence interval ( $\mathrm{Cl}$ ) were discovered by Cox proportional hazard model analysis. Statistically significant was regarded as the $P<0.05$.

\section{Results}

\subsection{Siglec10 expression in glioma patients with bioinformatics datasets}

The expression of siglec10 was discovered in different subtypes and grades of gliomas. As shown in Fig. 1 and Fig. 2, the siglec10 expression was higher in tumor tissue than normal tissue in LGG and GBM. Siglec10 expression in was higher in high grade gliomas (grade III, IV) than low grade gliomas (grade II). In addition, the expression of siglec10 was lower in IDH mutated gliomas than IDH wide-type gliomas. As for the different subtypes of gliomas, siglec10 expression was higher in mesenchymal subtype than classical, neural, and proneural subtypes.

In addition, bioinformatics datasets TCGA and CGGA were used to analyze the survival prognosis of glioma patients. High siglec10 expression patients had shorter survival prognosis than low siglec10 expression patients in TCGA dataset $(P=0.00058)$ and CGGA dataset $(P \otimes 0.0001)($ Fig. 1E, 1F).

\subsection{Higher siglec10 expression predicts worse survival in glioma patients}

To comfirm the results above, 162 samples of glioma patients from Sanbo Brain Hospital Capital Medical University were analyzed by immunohistochemical staining method. The results showed that high siglec10 expression patients had shorter survival prognosis than low siglec10 expression patients $(P=$ 0.00044 , Fig. 3A). Figure 3B displayed the clinical forest of siglec10 expression in glioma patients. High siglec10 expression had shorter survival prognosis than low siglec10 expression in patients with grade IV $(P=0.003), \operatorname{GBM}(P=0.003)$, ATRX loss $(P \otimes 0.001)$, no radiotherapy $(P=0.022)$, or no chemotherapy $(P=$ 0.016).

3.3. Siglec 10 was significant correlated with tumorigenic inflammatory cells, immune checkpoints and immune related genes

It has been reported that siglec10 was an innate immune checkpoint in macrophage and may as a potential target for immunetherapy in ovarian and breast cancer (13). Therefore, we mainly focused on its immune mechanism in glioma. Firstly, the correlation between siglec10 and inflammatory cells in tumor microenvironment was revealed. Inflammatory cells were clustered into three clusters with hclust method. In TCGA dataset, siglec10 was classfied into tumorigenic inflammatory cells including regulatory $T$ cells, myeloid-derived suppressor cells (MDSCs), macrophages, and immature dendritic cells (Cell cluster-A, Fig. 4A). In CGGA dataset, siglec10 was classfied into tumorigenic inflammatory cells including 
regulatory T cells, myeloid-derived suppressor cells (MDSCs), macrophages, plasmacytoid dendritic cells, and neutrophils (Cell cluster-A, Fig. 4B).

Secondly, the correlation between siglec10 and immune related genes was discovered by SSGSEA, either. Siglec10 was positively correlated with immune related genes including cytokines, chemokines, interferons, BCR signaling pathway, antigen processing and presentation, etc (Fig. 4C and 4D).

Furthermore, the relationship between siglec10 and other immune checkpoints was discovered. In TCGA dataset, siglec10 was correlated with programmed cell death 1 (PDCD1), cytotoxic T-lymphocyteassociated protein 4 (CTLA4), lymphocyte activating 3 (LAG3), T cell immunoreceptor with Ig and ITIM domains (TIGIT) (Fig. 4E). In CGGA dataset, siglec10 was correlated with PDCD1, CTLA4, LAG3, TIGIT, either (Fig. 4F).

\subsection{Siglec10 was associated with multiple immune-related signaling pathways}

We used GSEA analysis to investigate the related inflammatory pathways of siglec10 (Fig. 5). In TCGA dataset, siglec10 was related with IL6-JAK-STAT3 signaling pathway, reactive oxygen species, TGF $\beta$ signaling pathway. In CGGA dataset, siglec10 was related with IL6-JAK-STAT3 signaling pathway, reactive oxygen species, TGF $\beta$ signaling pathway, either.

\subsection{GO and KEGG analysis}

In this study, we used GO and KEGG analysis to conduct functional enrichment analysis. The data obtained from TCGA dataset were performed GO and KEGG analysis (Fig. 6A and 6B). GO analysis indicated the correlated genes of siglec 10 were enriched in neutrophil activation, neutrophil degranulation, neutrophil medicated immunity, T cell activation, regulation of lymphocyte activation, etc. KEGG signaling pathway analysis showed that the correlated genes of siglec 10 were related with tuberculosis, osteoclast differentiation, phagosome, staphylococcus aureus infection, cytokine-cytokine receptor interaction, etc.

Furthermore, the data obtain from CGGA dataset were performed GO and KEGG analysis (Fig. 6C and 6D). GO analysis indicated the correlated genes of siglec10 were enriched in neutrophil activation, neutrophil degranulation, neutrophil medicated immunity, $\mathrm{T}$ cell activation, immune response-regulating cell surface receptor signaling pathway, etc. KEGG signaling pathway analysis showed that the correlated genes of siglec10 were related with osteoclast differentiation, cytokine-cytokine receptor interaction, phagosome, tuberculosis, staphylococcus aureus infection, B cell receptor signaling pathway, etc.

\section{Discussion}

Siglec10 is a member of immunoglobulin superfamily expressed on the cell surface (15). Siglec10 plays important role in hepatocellular cancer, ovarian cancer, and triple-negative cancer. In the hepatocellular cancer, high siglec10 expression is correlated with reduced overall survival of hepatocellular cancer patients (12). In addition, siglec10 expression was higher in hepatocellular cancer tissue compared with 
adjacent normal tissue. The researcher considered siglec10 might reduce the anti-tumor function of natural killer cells to deteriorate hepatocellular cancer. In ovarian cancer and triple-negative cancer, tumor associated macrophages expressed high level of siglec10 (13). Down-regulated siglec10 expression could lead to the increased survival prognosis of ovarian cancer patients and triple-negative cancer patients. However, the expression and function of siglec10 in glioma was unknown. In this study, we investigated the expression and related mechanisms of siglec10 in glioma patients.

In this study, we discovered the siglec10 expression in glioma patients. In the results of immunohistochemical staining from glioma patients, we found high siglec10 expression patients had shorter survival prognosis than low siglec10 expression patients. From the TCGA and CGGA bioinformatics datasets, we also demonstrated found high siglec10 expression patients had shorter survival prognosis than low siglec 10 expression patients. Thus, we inferred siglec 10 was correlated with poor prognosis survival in gliomas. Then, we studied the clinical features of siglec10 expression in glioma patients. High siglec10 expression had shorter survival prognosis than low siglec10 expression in patients with grade IV, GBM, ATRX loss, no radiotherapy, or no chemotherapy. Grade IV belongs to high grade gliomas and high grade gliomas are malignant with poor survival prognosis (16). GBM is one of most aggressive cancers that the survival duration of GBM after diagnosis is only 12 to 15 months (17). ATRX is a biomarker of glioma molecular classification (18). ATRX deficiency is correlated with poor survival prognosis of glioma patients. No radiotherapy and chemotherapy are also related with poor survival prognosis in gliomas. Grade IV, GBM, ATRX loss, no radiotherapy, and no chemotherapy are all malignant factors in gliomas. So, high siglec10 expression is related with short survival prognosis especially in patients with poor survival prognosis. Siglec10 might correlate with other malignant factors in gliomas.

In addition, we studied the siglec10 expression in different grades and subtypes of gliomas. The siglec10 expression is higher in tumor tissue than normal tissue. In addition, siglec10 expression is higher in high grade gliomas than low grade gliomas. Furthermore, siglec10 expression is highest in mesenchymal subtype than classical, neural, and proneural subtypes. Compared with other three glioma subtypes, mesenchymal subtype has worst survival prognosis (19). So, we inferred siglec10 contributes to the poor prognosis and therapy resistance of gliomas.

Then, we investigated the possible mechanisms about siglec10 in gliomas. The results showed siglec10 was correlated with inflammatory response in the tumor microenvironment of gliomas. In the results of sSGSEA, siglec10 was related with tumorigenic inflammatory cell infiltration including MDSCs, macrophages, regulatory T cells, etc. The immune related genes included cytokines, chemokines, interferons, etc. In the results of GSEA, siglec10 was related with inflammatory pathways. In the results of GO and KEGG analysis, correlated genes of siglec10 were enriched neutrophil immune response, lymphocyte activation, etc. Inflammation might have deleterious effects at tumor growth, tissue injury, or infection. In some types of cancers, inflammation leads to tumorigenesis by activating reactive oxygen and nitrogen species secreted by neutrophils and macrophages (20). Gliomas produce inflammatory and proangiogenic microenvironment leading to reduced tight junction of blood-brain barrier. Activated 
microglias increase the levels of major histocompatibility complex class II (MHCII) to enhance the ability of antigen processing and presentation (21). Then, pro-inflammatory mediators including cytokines, chemokines, and interferons are produced to accelerate inflammation. The inflammatory mediators could regulate blood-brain barrier to induce bone marrow-derived immune cells into central nervous system including neutrophils, macrophages, dendritic cells, T cells, etc.

The siglec10 related inflammatory cells were MDSCs, macrophages, neutrophils. MDSCs are robustly recruited in the tumor microenvironment of gliomas. MDSCs induce tumor metastasis and angiogenesis by secreting inflammatory mediators including cytokines, matrix metalloproteinases (MMPs), fibroblast growth factor, and vascular endothelial growth factor (VEGF). $(22,23)$ MMPs produced by MDSCs play critical role in matrix degradation (24). VEGF produced by MDSCs are helpful to produce pre-metastatic environment in gliomas. Tumor associated macrophages (TAMs) consist approximately $40 \%$ immune cells population in glioma patients (25). TAMs interact with tumor cells in TME to induce tumor growth and metastasis (26). TAMs could produce pro-inflammatory mediators including TNF-a, IL-6, IL-12 to amplify inflammation in gliomas(27). In addition, TAMs up-regulate the expression of cell surface molecules and co-stimulatory molecules to facilitate antigen processing and presentation. Tumorassociated neutrophils (TANs) are recruited and stimulated by inflammatory mediators in TME (28). The infiltration of neutrophils was related with glioma grades. Neutrophils induce the phenotypes of malignant glioma in anti-VEGF therapy (29). Apart from inflammatory cells, we discovered that siglec10 was correlated with immune checkpoints. Immune checkpoints were inhibitory factors to prevent immune response in immune system (30). They were overexpressed in the tumor microenvironment of gliomas to exert tumorgenesis effects (31). The immune checkpoints such as CTLA-4 could down-regulate the activation of cytotoxic T lymphocytes in the tumor microenvironment (32). Siglec10 was found to be correlated with immune checkpoints in our study. Thus, we inferred that siglec10 possessed synergic action with immune checkpoints to exert tumorgenesis effects in gliomas.

\section{Conclusion}

In this study, we revealed the expression and mechanisms of siglec10 in gliomas. From the results of immunohistochemical staining and bioinformatics datasets, we found that high siglec10 expression was correlated with short survival prognosis in gliomas especially in malignant patients. In addition, the expression of siglec 10 was higher in malignant subtype than benign subtype of gliomas. Thus, we inferred siglec10 contributes to the poor prognosis and therapy resistance of glioma patients.

Furthermore, the related mechanisms of siglec10 were investigated by GSEA, GO, and KEGG analysis. The results indicated siglec10 was correlated with tumorigenesis inflammatory cells in gliomas. Siglec10 was also correlated with immune checkpoints in gliomas. This study showed siglec 10 was a biomarker in glioma and it might be the target of glioma immunotherapy in the future.

\section{Declarations}

\section{Ethics approval and consent to participate}


The Ethical Committee and Institutional Review Board of the Sanbo Brain Hospital Capital Medical University reviewed and approved this study protocol. All patients signed written informed consent.

\section{Consent for publication}

Not applicable.

\section{Availability of data and materials}

The data that support the findings of this study are available from the corresponding author.

\section{Competing interests}

The authors declare no conflict of interest.

\section{Funding}

This work was supported by the National Natural Science Foundation of China [grant numbers 81904142], Projects of International Cooperation and Exchanges [grant number 2014DFA32950].

\section{Authors' contributions}

Y.F., Y.Z. performed statistical analysis, and drafted the manuscript. T.W., Y.F. H.W., contributed to database building. K.Y. conceived the design of the study and revised the manuscript. All authors read and approved the final manuscript.

\section{Acknowledgements}

Not applicable

\section{References}

1. Ostrom QT, Gittleman H, Stetson L, et al. Epidemiology of gliomas. Cancer Treat Res. 2015;163:1-14.

2. Davis ME. Epidemiology and Overview of Gliomas. Semin Oncol Nurs. 2018;34(5):420-9.

3. Perry A, Wesseling P. Histologic classification of gliomas. Handb Clin Neurol. 2016;134:71-95.

4. Molinaro AM, Taylor JW, Wiencke JK, et al. Genetic and molecular epidemiology of adult diffuse glioma. Nat Rev Neurol. 2019;15(7):405-17.

5. Siegal T. Clinical Relevance of Prognostic and Predictive Molecular Markers in Gliomas. Adv Tech Stand Neurosurg. 2016;(43):91-108.

6. Turkalp Z, Karamchandani J, Das S. IDH mutation in glioma: new insights and promises for the future. JAMA Neurol. 2014;71(10):1319-25.

7. Cankovic M, Nikiforova MN, Snuderl M, et al. The role of MGMT testing in clinical practice: a report of the association for molecular pathology. J Mol Diagn. 2013;15(5):539-55. 
8. Binabaj MM, Bahrami A, ShahidSales S, et al. The prognostic value of MGMT promoter methylation in glioblastoma: A meta-analysis of clinical trials. J Cell Physiol. 2018;233(1):378-86.

9. Li N, Zhang W, Wan T, et al. Cloning and characterization of Siglec-10, a novel sialic acid binding member of the Ig superfamily, from human dendritic cells. J Biol Chem. 2001;276(30):28106-12.

10. Shathili AM, Bandala-Sanchez E, John A, et al. Specific Sialoforms Required for the Immune Suppressive Activity of Human Soluble CD52. Front Immunol. 2019;10:1967.

11. Kivi E, Elima K, Aalto K, et al. Human Siglec-10 can bind to vascular adhesion protein-1 and serves as its substrate. Blood. 2009;114(26):5385-92.

12. Zhang $P$, Lu $X$, Tao $K$, et al. Siglec-10 is associated with survival and natural killer cell dysfunction in hepatocellular carcinoma. J Surg Res. 2015;194(1):107-13.

13. Barkal AA, Brewer RE, Markovic M, et al. CD24 signalling through macrophage Siglec-10 is a target for cancer immunotherapy. Nature. 2019;572(7769):392-6.

14. Bandala-Sanchez E, Bediaga NG, Naselli G, et al. Siglec-10 expression is up-regulated in activated human $\mathrm{CD} 4^{+} \mathrm{T}$ cells. Hum Immunol. 2020;81(2-3):101-4.

15. Munday J, Kerr S, Ni J, et al. Identification, characterization and leucocyte expression of Siglec-10, a novel human sialic acid-binding receptor. Biochem J. 2001;355(Pt 2):489-97.

16. Nayak L, Reardon DA. High-grade Gliomas. Continuum (Minneap Minn). 2017;23(6):1548-63.

17. Omuro A, DeAngelis LM. Glioblastoma and other malignant gliomas: a clinical review. JAMA. 2013;310(17):1842-50.

18. Nandakumar P, Mansouri A, Das S. The Role of ATRX in Glioma Biology. Front Oncol. 2017;7:236.

19. Jakovlevs A, Vanags A, Gardovskis J, et al. Molecular classification of diffuse gliomas. Pol J Pathol. 2019;70(4):246-58.

20. Greten FR, Grivennikov SI. Inflammation and Cancer: Triggers, Mechanisms, and Consequences. Immunity. 2019;51(1):27-41.

21. Qian J, Luo F, Yang J, et al. TLR2 Promotes Glioma Immune Evasion by Downregulating MHC Class II Molecules in Microglia. Cancer Immunol Res. 2018;6(10):1220-33.

22. Umansky V, Blattner C, Gebhardt C, et al. The Role of Myeloid-Derived Suppressor Cells (MDSC) in Cancer Progression. Vaccines (Basel). 2016;4(4). pii: E36.

23. Ugel S, De Sanctis F, Mandruzzato S, et al. Tumor-induced myeloid deviation: when myeloid-derived suppressor cells meet tumor-associated macrophages. J Clin Invest. 2015;125(9):3365-76.

24. Du R, Lu KV, Petritsch C, et al. HIF1alpha induces the recruitment of bone marrow-derived vascular modulatory cells to regulate tumor angiogenesis and invasion. Cancer Cell. 2008;13(3):206-20.

25. Gieryng A, Pszczolkowska D, Walentynowicz KA, et al. Immune microenvironment of gliomas. Lab Invest. 2017;97(5):498-518.

26. Sevenich L. Brain-Resident Microglia and Blood-Borne Macrophages Orchestrate Central Nervous System Inflammation in Neurodegenerative Disorders and Brain Cancer. Front Immunol. 2018;9:697. 
27. Zhai H, Heppner FL, Tsirka SE. Microglia/macrophages promote glioma progression. Glia. 2011;59(3):472-85.

28. Shaul ME, Fridlender ZG. Tumour-associated neutrophils in patients with cancer. Nat Rev Clin Oncol. 2019;16(10):601-20.

29. Liang J, Piao Y, Holmes L, et al. Neutrophils promote the malignant glioma phenotype through S100A4. Clin Cancer Res. 2014;20(1):187-98.

30. Chen L, Flies DB. Molecular mechanisms of T cell co-stimulation and co-inhibition. Nat Rev Immunol. 2013;13(4):227-42.

31. Topalian SL, Drake CG, Pardoll DM. Immune checkpoint blockade: a common denominator approach to cancer therapy. Cancer Cell. 2015;27(4):450-61.

32. Yang Y. Cancer immunotherapy: harnessing the immune system to battle cancer. J Clin Invest. 2015;125(9):3335-7.

\section{Figures}


A

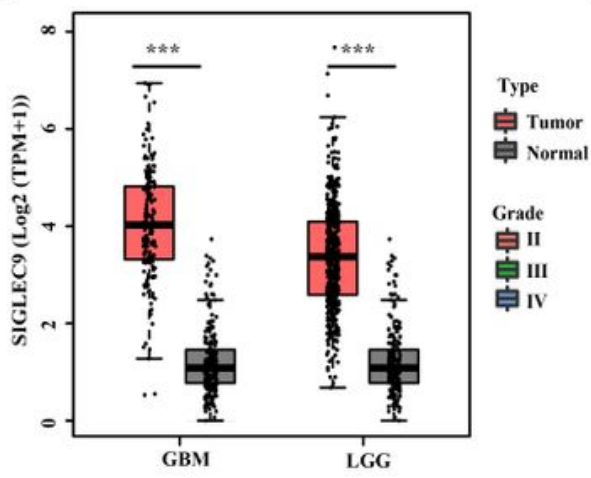

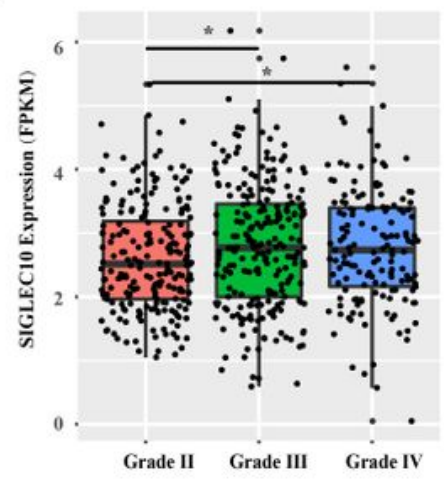

C

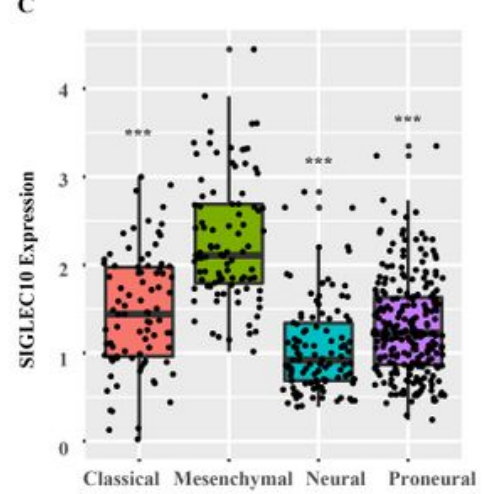

D
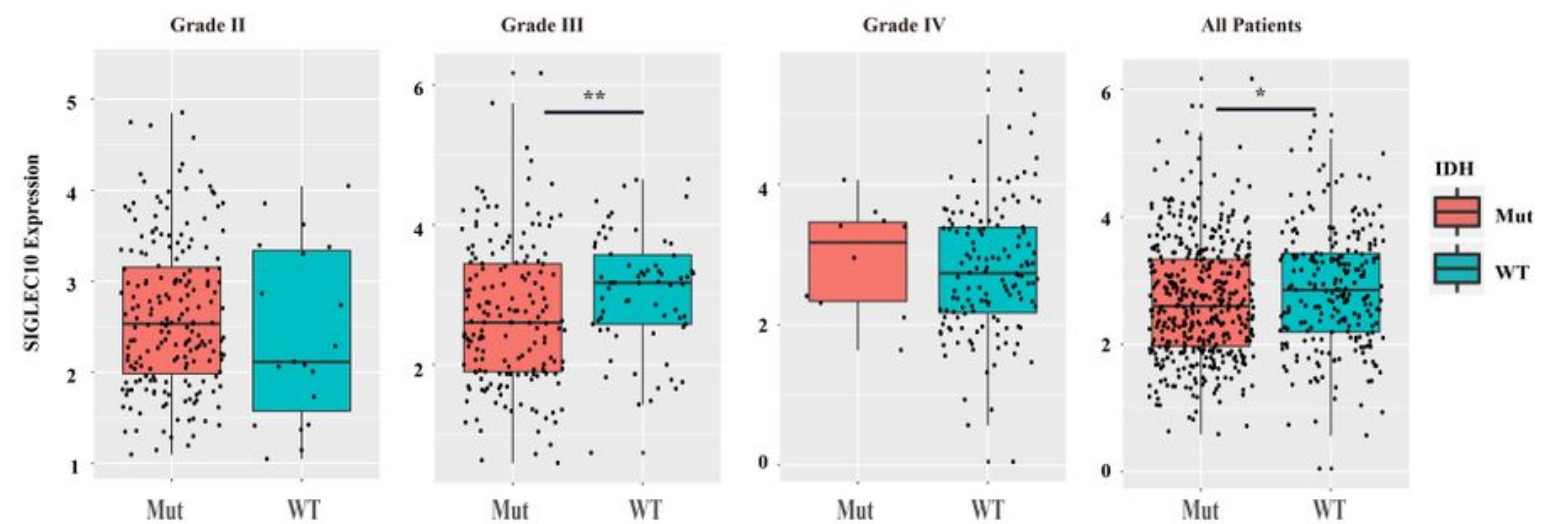

E
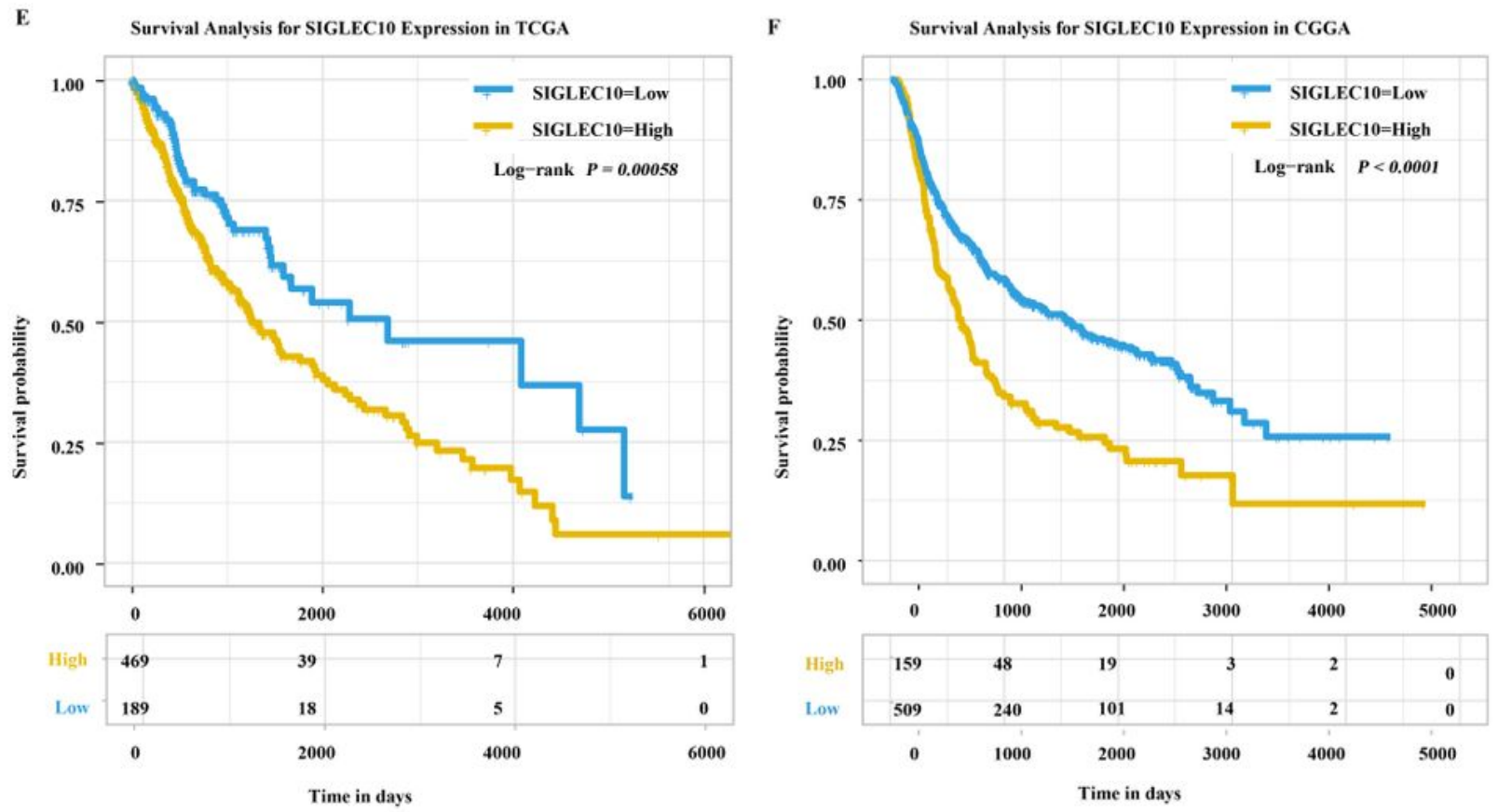

\section{Figure 1}

The expression of siglec10 in different subtypes and grades of gliomas. (A) In TCGA dataset, siglec10 expression was higher in tumor tissue than normal tissue in LGG and GBM (*represented P $\otimes 0.05$ ). (B) In TCGA dataset, siglec10 expression was higher in high grade gliomas than low grade gliomas (*represented $P \otimes 0.05$ ). (C) In TCGA dataset, siglec10 expression was higher in mesenchymal subtype than classical, neural, and proneural subtypes (**represented P $\otimes 0.01$ ). (D) In TCGA dataset, siglec10 
expression was higher in IDH mutated gliomas than IDH wide-type gliomas (*represented $\mathrm{P} \otimes 0.05$; $\star \star$ represented $P \otimes 0.01)$. (E) High siglec10 expression patients had shorter survival prognosis than low siglec10 expression patients in TCGA dataset $(P=0.00058)$. $(F)$ High siglec10 expression patients had shorter survival prognosis than low siglec10 expression patients in CGGA dataset $(P \otimes 0.0001)$.
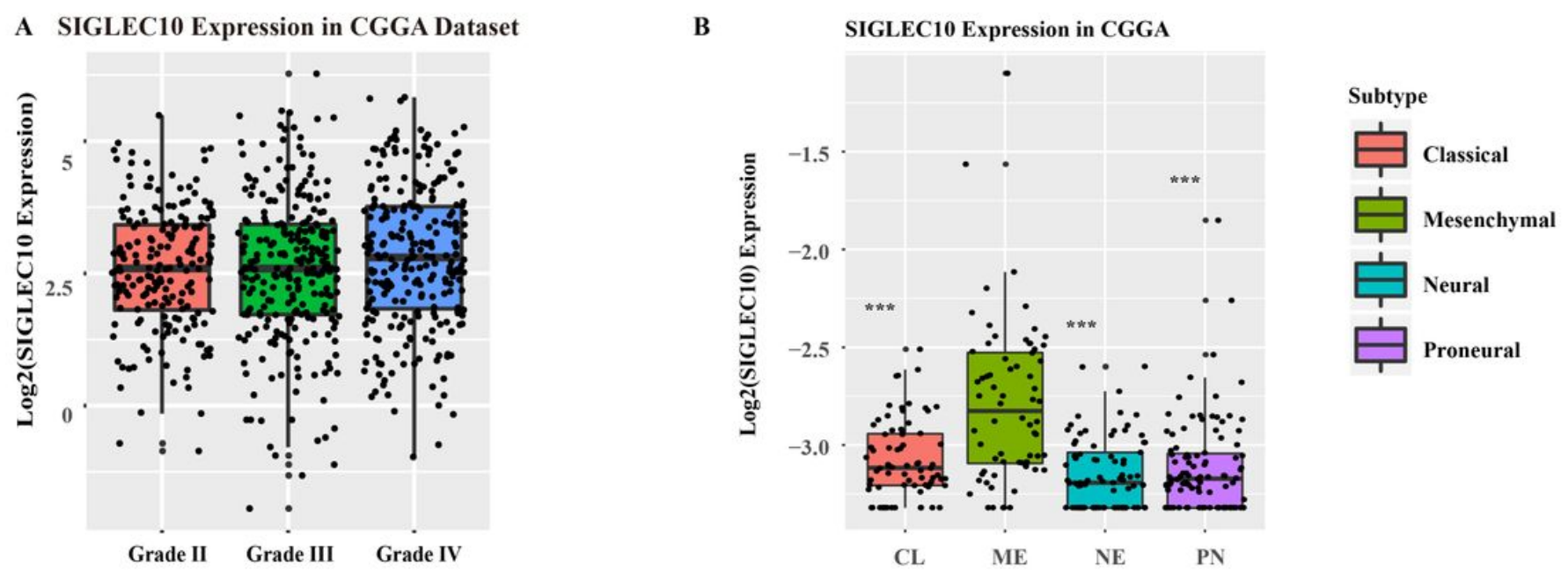

C
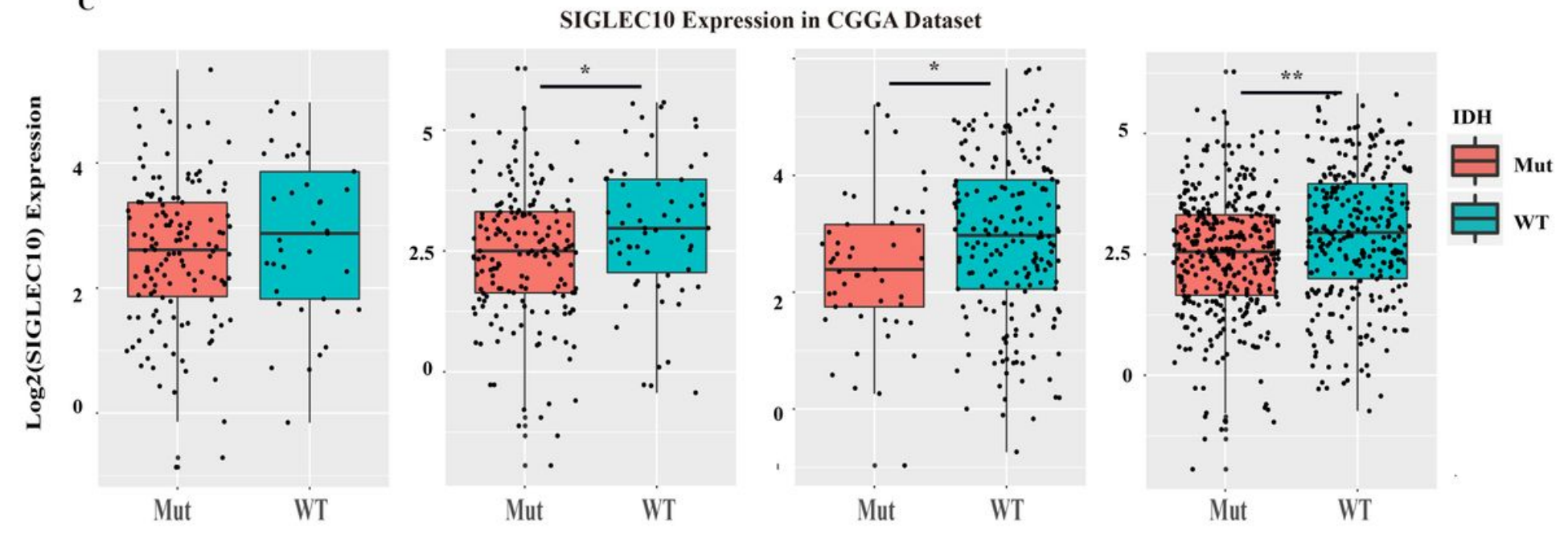

Figure 2

Siglec10 expression in glioma patients with CGGA dataset. (A) In CGGA dataset, siglec10 expression in was higher in high grade gliomas than low grade gliomas. (B) In CGGA dataset, siglec10 expression was higher in mesenchymal subtype than classical, neural, and proneural subtypes (**represented $P \otimes 0.01$ ).

(C) In CGGA dataset, siglec10 expression was higher in IDH mutated gliomas than IDH wide-type gliomas (*represented $\mathrm{P} \otimes 0.05$; **represented $\mathrm{P} \otimes 0.01$ ). 


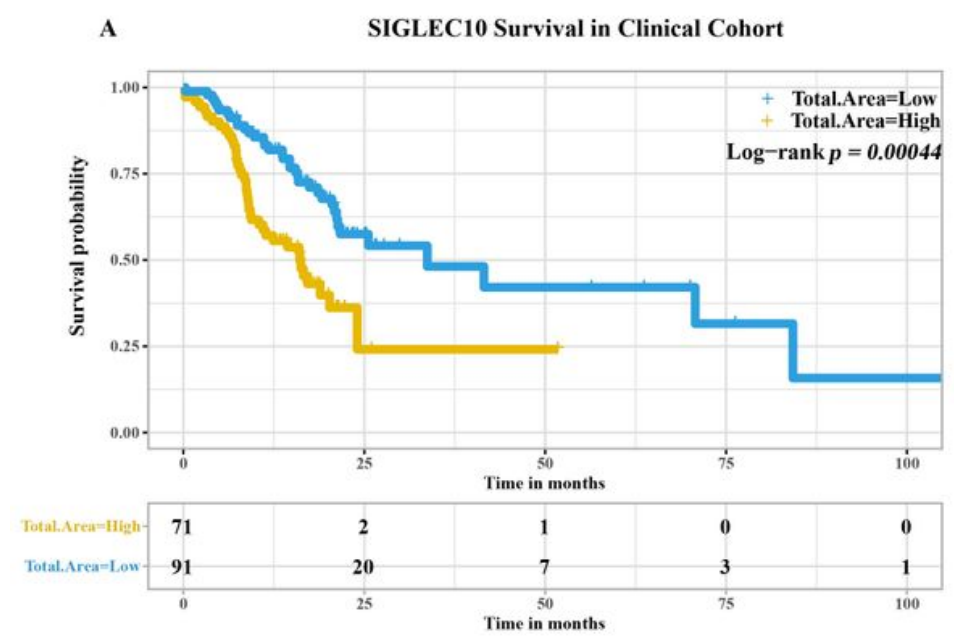

C

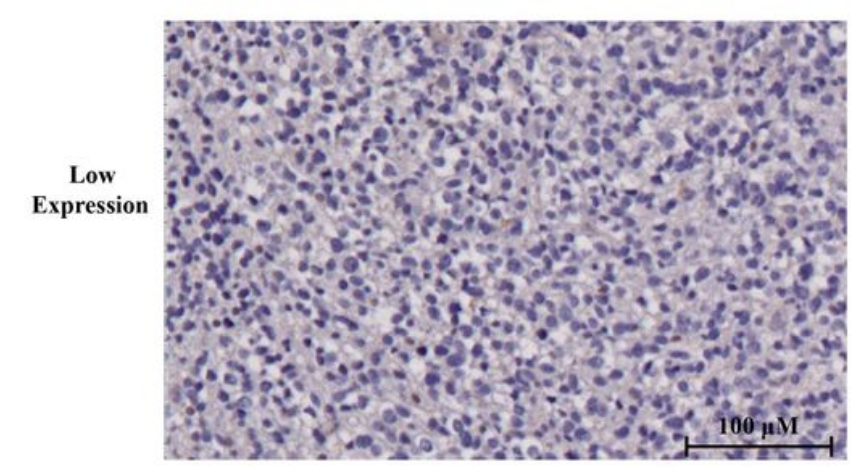

B

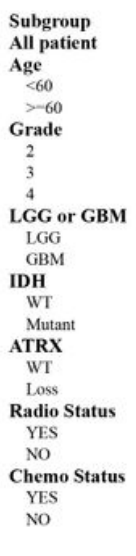

Clinical Forest
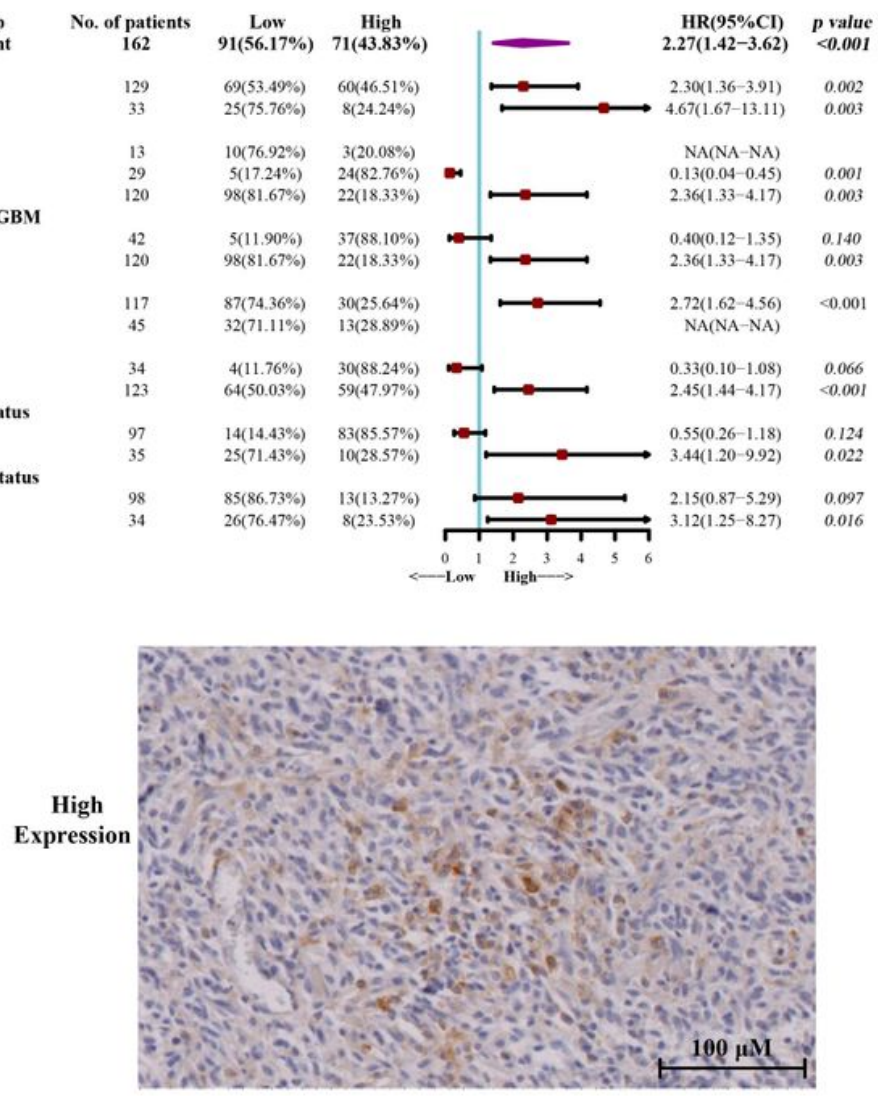

\section{Figure 3}

Siglec10 expression in glioma patients. (A) Siglec10 survival in clinical cohort with immunohistochemical staining method. High siglec10 expression patients had shorter survival prognosis than low siglec10 expression patients $(P \otimes 0.001)$. (B) Clinical forest of the cohort. High siglec10 expression had shorter survival prognosis than low siglec10 expression in patients with grade IV $(P=0.003), G B M(P=0.003)$, ATRX loss $(P \otimes 0.001)$, no radiotherapy $(P=0.022)$, or no chemotherapy $(P=0.016)$. (C) Immunohistochemical staining of SIGLEC10 expression. 

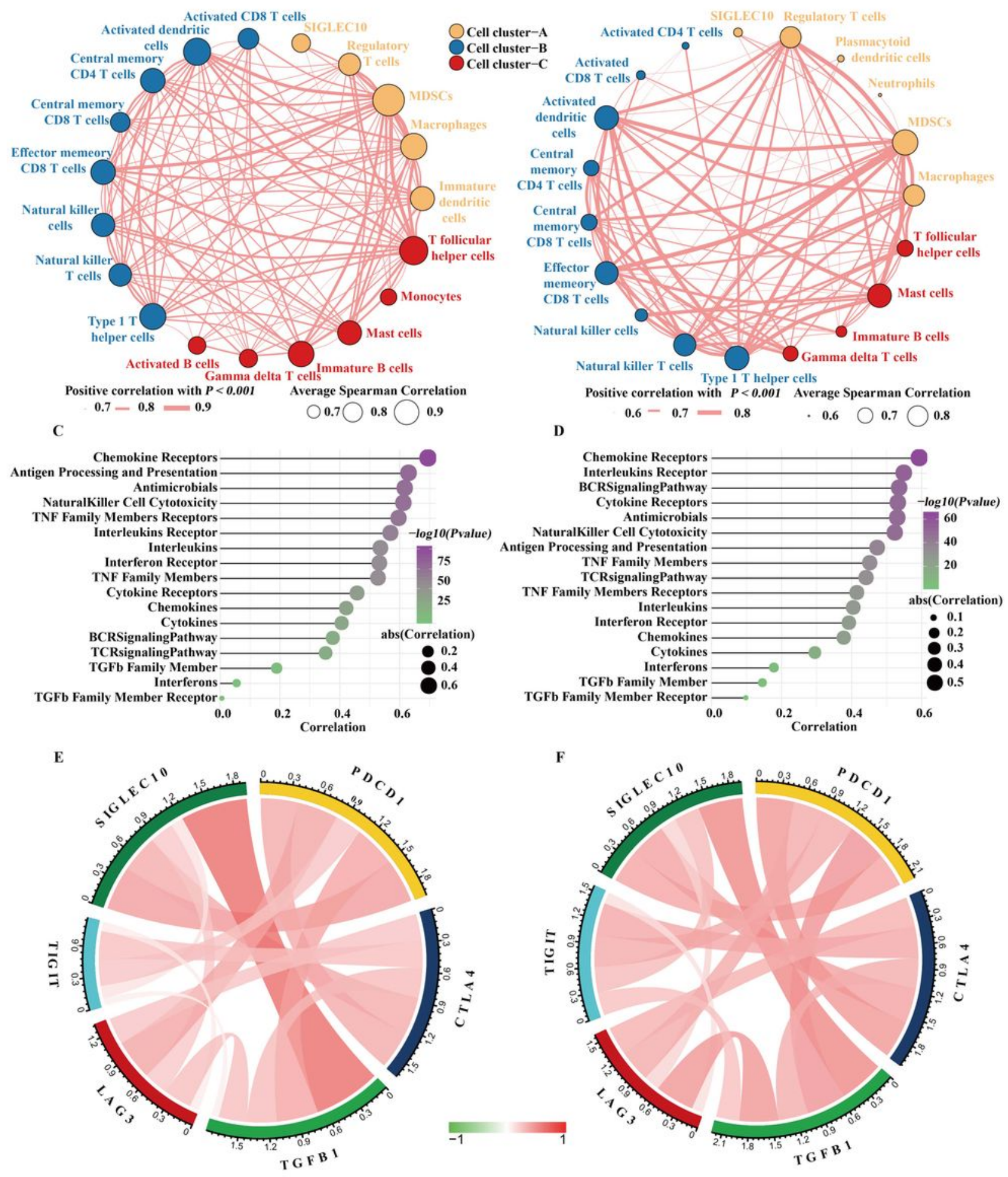

\section{Figure 4}

The correlation between siglec10 and inflammatory status. (A) The spearman correlation between siglec10 and inflammatory cells in TCGA dataset. The correlation value $>0.7$ were used. (B) The spearman correlation between siglec10 and inflammatory cells in CGGA dataset.The correlation value > 0.6 were used. (C) The correlation between siglec10 and inflammatory related genes in TCGA dataset. (D) The correlation between siglec10 and inflammatory related genes in CGGA dataset. (E) The relationship 
A

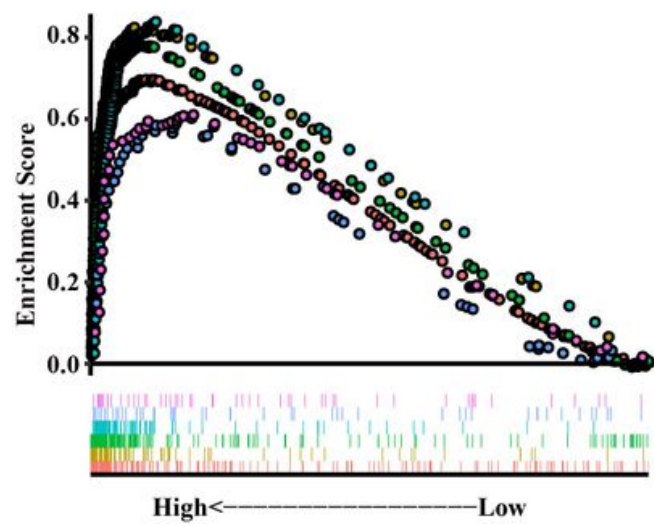

B

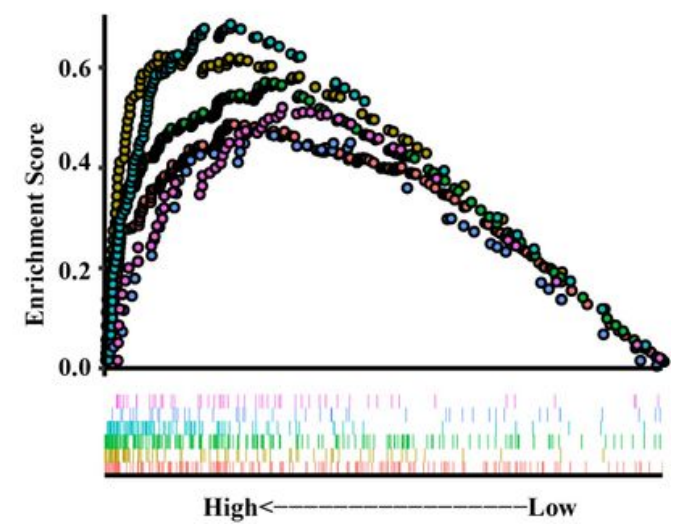

Figure 5

The related inflammatory pathways of siglec10 with GSEA analysis. (A) The related inflammatory pathways of siglec10 from TCGA dataset with GSEA analysis. (B) The related inflammatory pathways of siglec10 from CGGA dataset with GSEA analysis.

A

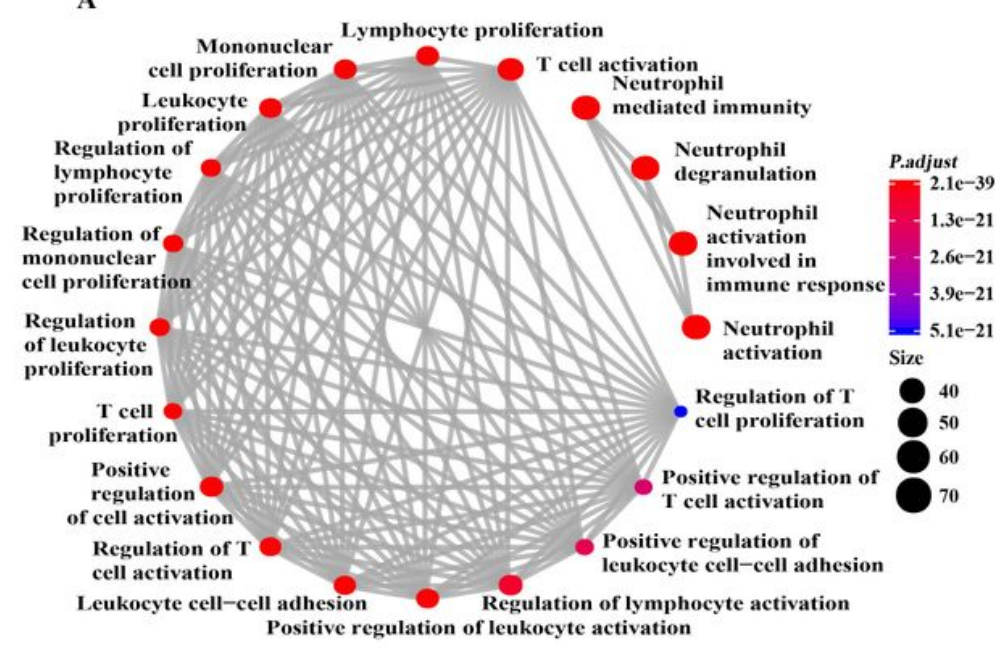

C

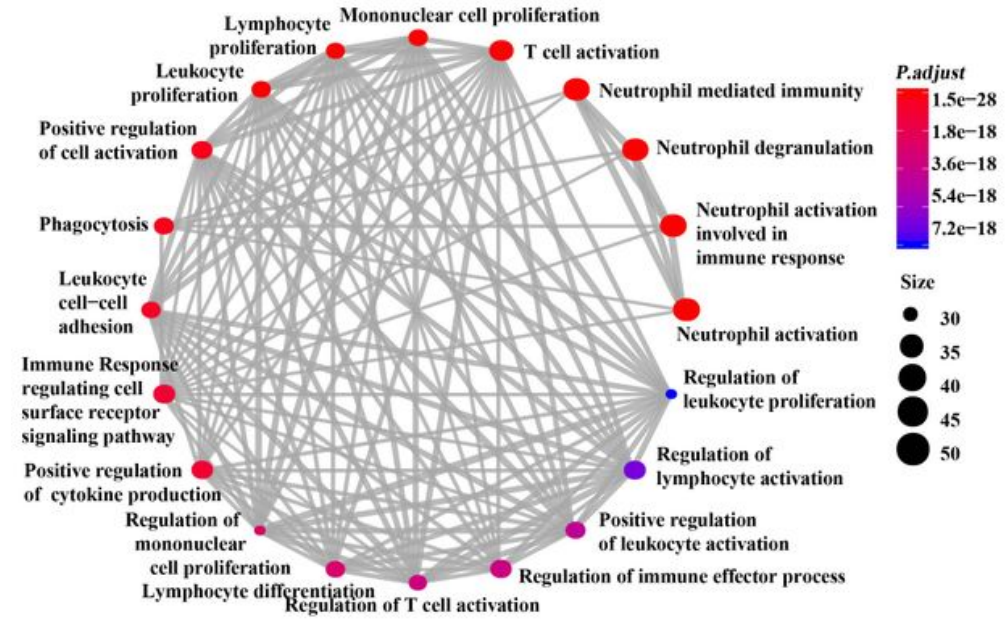

B

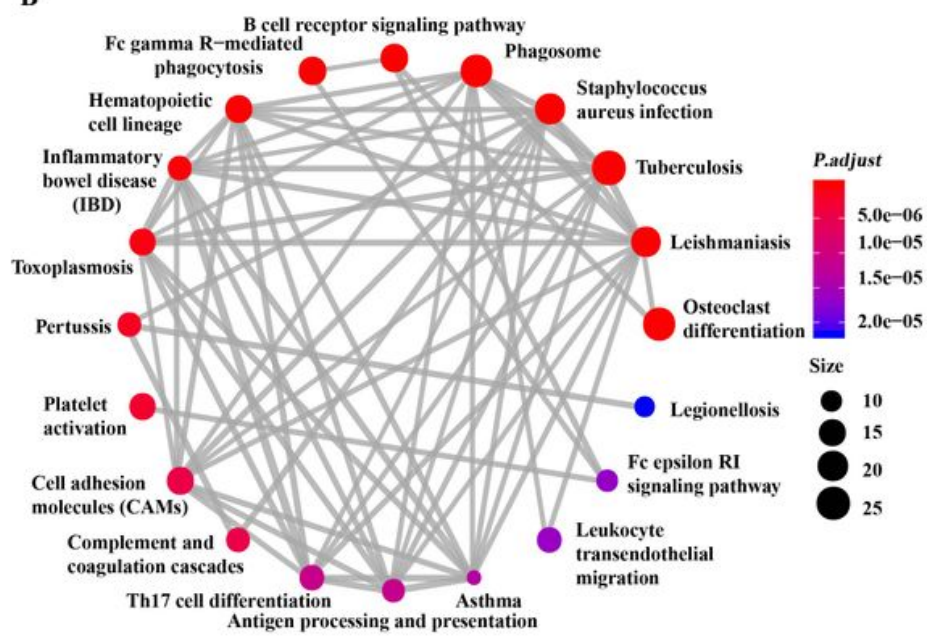

D

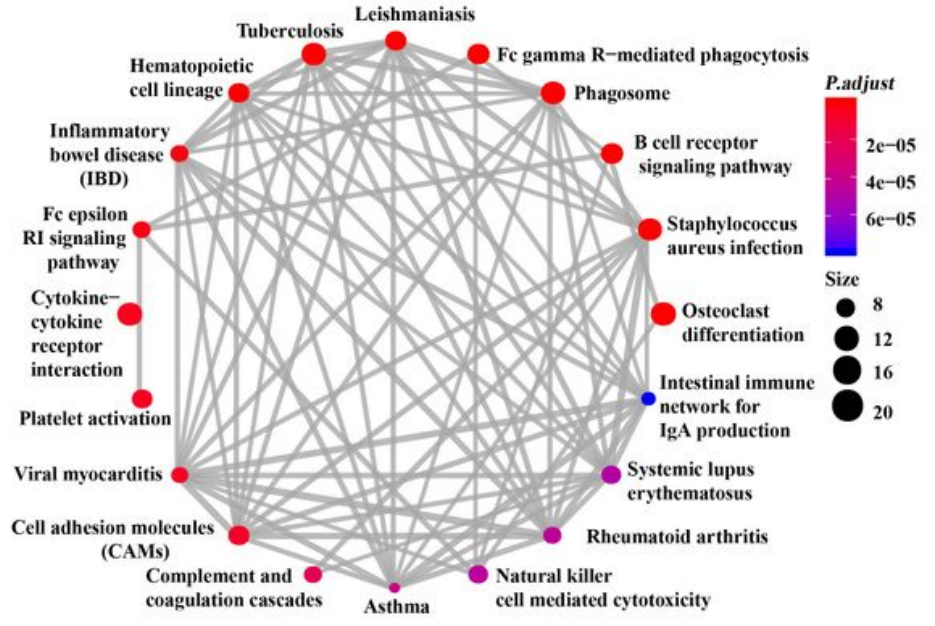


Figure 6

GO and KEGG signaling pathway analysis of siglec10 related genes. (A) GO analysis of siglec10 related genes with TCGA dataset. (B) KEGG signaling pathway analysis of siglec10 related genes with TCGA dataset. (C) GO analysis of siglec10 related genes with CGGA dataset. (D) KEGG signaling pathway analysis of siglec10 related genes with CGGA dataset. 